\title{
Determination of the top quark mass circa 2013: methods, subtleties, perspectives
}

\author{
Aurelio Juste ${ }^{1,2}$, Sonny Mantry ${ }^{3,4}$, Alexander Mitov ${ }^{5, a}$, Alexander Penin ${ }^{6,7}$, Peter Skands ${ }^{5}$, Erich Varnes ${ }^{8}$, \\ Marcel Vos ${ }^{9}$, Stephen Wimpenny ${ }^{10}$ \\ ${ }^{1}$ Institució Catalana de Recerca i Estudis Avançats (ICREA), 08010 Barcelona, Spain \\ ${ }^{2}$ Institut de Física d'Altes Energies, Universitat Autonòma de Barcelona, 08193 Bellaterra, Spain \\ ${ }^{3}$ High Energy Division, Argonne National Laboratory, Argonne, IL 60439, USA \\ ${ }^{4}$ Department of Physics and Astronomy, Northwestern University, Evanston, IL 60208, USA \\ 5 Theory Division, CERN, 1211 Geneva 23, Switzerland \\ ${ }^{6}$ University of Alberta, Edmonton, AB T6G 2J1, Canada \\ ${ }^{7}$ Institut für Theoretische Teilchenphysik, KIT, 76128 Karlsruhe, Germany \\ ${ }^{8}$ Department of Physics, University of Arizona, Tucson, AZ 85721, USA \\ ${ }^{9}$ IFIC (UVEG/CSIC), Ap. Correos 22085, 46071 Valencia, Spain \\ ${ }^{10}$ Department of Physics and Astronomy, University of California, Riverside, Riverside, CA 92521, USA
}

Received: 19 October 2013 / Accepted: 7 October 2014 / Published online: 29 October 2014

(C) The Author(s) 2014. This article is published with open access at Springerlink.com

\begin{abstract}
We present an up-to-date overview of the problem of top quark mass determination. We assess the need for precision in the top mass extraction in the LHC era together with the main theoretical and experimental issues arising in precision top mass determination. We collect and document existing results on top mass determination at hadron colliders and map the prospects for future precision top mass determination at $e^{+} e^{-}$colliders. We present a collection of estimates for the ultimate precision of various methods for top quark mass extraction at the LHC.
\end{abstract}

\section{Introduction}

The precision with which we determine the top quark mass impacts our understanding of several phenomena. Examples are EW precision fits [1], determination of the vacuum stability in the Standard Model [2,3] as well as models with broad cosmological implications $[4,5]$.

A number of measurements of $m_{t}$ from hadron colliders exist $[6,7]$, utilizing all measured decay modes of the top quark. The experimental extraction has accuracy of $\delta m_{t} \leq$ $1 \mathrm{GeV}$. The main task for this writeup is to map the steps that can clarify the relation between the extracted value of

Preprint number: CERN-PH-TH/2013-226.

a e-mail: adm74@cam.ac.uk the mass and a theoretically well-defined top mass (like the pole mass).

The top quark mass $m_{t}$ is not a physical observable and, therefore, it cannot be measured directly. Virtually all existing strategies for determining $m_{t}$ (see Sect. 3) are based on its extraction from observables that are directly sensitive to it, i.e. $m_{t}$ is defined as the solution to the following implicit relation:

$\sigma^{\exp }(\{Q\})=\sigma^{\text {th }}\left(m_{t},\{Q\}\right)$,

where $\{Q\}$ is a set of kinematical variables, $\sigma^{\exp }$ stands for the measured and $\sigma^{\text {th }}$ for the predicted value of some chosen observable $\sigma$. In a typical application, $m_{t}$ is adjusted in $\sigma^{\text {th }}$ to obtain the best fit to the shape of $\sigma^{\exp }$, as a function of the variables $\{Q\}$. This implicitly assumes that $\sigma^{\exp }$ has been corrected for detector (and possibly acceptance) effects, or that the converse has been applied to $\sigma^{\text {th }}$, so that the observables on either side of Eq. 1 are defined at the same level, with the same cuts. Uncertainties in the theoretical prediction due to missing higher-order effects, finite-width effects, and non-perturbative corrections are generally present. We discuss them in more detail in Sect. 2.

The top mass $m_{t}$ is scheme dependent and a large number of such schemes exist. Examples are the pole, the $\overline{\mathrm{MS}}$, and the threshold mass schemes [8-10]; see Refs. [11,12] for a discussion in the context of hadron colliders. Different mass schemes are perturbatively related to each other. For example, the top mass $m_{t}(R, \mu)$ in scheme " $R$ " is related to the pole mass $m_{t}^{\text {pole }}$ through a perturbative series 
$m_{t}^{\text {pole }}=m_{t}(R, \mu)+\delta m_{t}(R, \mu)$,

$\delta m_{t}(R, \mu)=R \sum_{n=1}^{\infty} \sum_{k=0}^{n} a_{n k}\left[\alpha_{s}(\mu)\right]^{n} \ln ^{k}\left(\frac{\mu^{2}}{R^{2}}\right)$,

where $R$ is a scale associated with the scheme; for the $\overline{\mathrm{MS}}$ scheme $R \sim m_{t}$. The relation between the pole and $\overline{\mathrm{MS}}$ masses is known to three loops in QCD [13,14]; a possible large EW correction has recently been reported in Ref. [15]. Large logarithms can arise in converting between schemes if the scale $R \ll m_{t}$, as seen in top resonance schemes [16] where $R \sim \Gamma_{\text {top }}$, and can be resummed via an infrared renormalization group equation $[17,18]$.

A reliable interpretation of top mass measurements requires understanding the connection between the theory prediction, in a given top mass scheme, and the experimental observable as shown schematically in Eq. (1). This connection between experimental observables and the appropriate top mass schemes is well understood in $e^{+} e^{-}$colliders. Precision top quark mass determinations at $e^{+} e^{-}$colliders have been studied for top pair production near threshold [19-22] and in the boosted regime [16,23]. In the boosted regime, a new class of top resonance mass schemes were introduced; additional top resonance mass definitions were given and explored in further detail in Ref. [24]. The expected uncertainty in the top $\overline{\mathrm{MS}}$ mass from the threshold scan method is $\delta m_{t} \leq 100 \mathrm{MeV}[25,26]$ and a few hundred MeV [27] for boosted top quarks. See Sects. 4, 4.1, and 4.4 for details.

The introduction of process-specific top mass definitions does not automatically solve the problem of top mass determination. If the perturbative relation between a processspecific mass and another "universal" mass (like the pole or $\overline{\mathrm{MS}}$ mass) is not known with sufficiently high accuracy, or does not converge well, then even a very precise determination of the value of this particular process-specific mass cannot be used in other processes. On the other hand, if the relation is precisely known and convergent, then effectively one is using a single mass definition in all processes.

For hadron colliders, which are the main focus of the current and near future research, and by extension of this document, the situation is more complicated and a rigorous framework is still lacking. Below we review the current status and issues in precision top mass extractions at hadron colliders. Unless explicitly specified, when in the following we refer to the top mass we mean the top pole mass and its associated total uncertainty.

\section{Issues in precision top mass determination at hadron colliders}

A unique property of the top quark is that it decays very quickly, before it can form strongly interacting bound states.
For this reason the top quark can be studied largely free of non-perturbative effects [19-21]. Still, a number of uncertainties of perturbative and non-perturbative origin affect the extraction of $m_{t}$ :

1. MC modeling Most methods for extraction of $m_{t}$ rely on modeling the measured final state with typically LO + LL MC generators. The extracted mass then reflects the mass parameter in the corresponding MC generator. Identifying the nature of this mass parameter and relating it to common mass schemes, like the pole mass, is a nontrivial and open problem, and it may be associated with ambiguities of order $1 \mathrm{GeV}$ [28, Appendix C]; see also Ref. [11]. The effect of the top and bottom masses on parton-shower radiation patterns is generally included already in the LO+LL Monte Carlos [29-34] and acts to screen the collinear singularities. NLO matching and non-perturbative effects are discussed separately below.

2. Reconstruction of the top pair Typically, the existing methods for extraction of the top quark mass implicitly or explicitly rely on the reconstruction of the top pair from final state leptons and jets. This introduces uncertainties of both perturbative origin (through higher-order corrections) and non-perturbative origin (related to hadronization and non-factorizable corrections). Methods that do not rely on such a reconstruction are therefore complementary and highly desirable; two examples are given in 3.4 and 3.5 .

3. Unstable top and finite top width effects These effects have been studied extensively in the context of top pair production at $e^{+} e^{-}$colliders [35-37]. In the context of higher-order corrections at hadron colliders, finite top (and $W$ ) width effects have been computed in [38-40] where comparisons versus the narrow width approximation can be found. The conclusion is that these corrections are small, sub-1\%, in inclusive observables (like the total inclusive cross section used in 3.3) but can be sizable in tails of kinematical distributions. In particular, they significantly affect the tail of the $B \ell$ invariant mass distribution used in the method 3.4 (but not the central region of the distribution which is most relevant for the $m_{t}$ determination described in 3.4).

4. Bound-state effects in top pair production at hadron colliders The effect of bound-state formation on top pair production at hadron colliders has been studied in Refs. [4144]. Despite the very short lifetime of the top quark, top-antitop bound-state formation starts to take place and this process affects the shape of differential distributions within a few $\mathrm{GeV}$ of absolute threshold. Therefore, any mass measurement that is sensitive to this kinematical region has to properly take these effects into consideration. In the context of the total cross section, see Refs. $[45,46]$, the effect on the cross section is sub- $1 \%$ 
and is taken into account in current higher-order calculations of the total inclusive cross section (and thus in mass extractions based upon it).

5. Renormalon ambiguity in top mass definition It is well known [17,47-49] that the pole mass of the top quark suffers from the so-called renormalon ambiguity. This implies an additional irreducible uncertainty of several hundred MeV's on the top pole mass. The short-distance masses do not suffer from the renormalon ambiguity and the precision in their determination is restricted only by experimental and theoretical uncertainties. At hadron colliders, where currently $\delta m_{t} \leq 1 \mathrm{GeV}$, the renormalon ambiguity is numerically subdominant; see also Ref. [12].

6. Alternative top mass definitions It is well understood from $e^{+} e^{-}$collider studies that by using alternative top mass definitions one could improve the precision of the extracted top quark mass. Similar studies for hadron colliders have been done in Refs. [11,12,50]. It has been argued in Ref. [11] that for top mass extractions in the peak region, the appropriate short-distance mass schemes correspond to the top resonance schemes where $R \sim R_{\mathrm{sc}} \sim 1 \mathrm{GeV} \sim \Gamma_{\text {top }}$, where $R_{\mathrm{sc}}$ is the shower cutoff implemented in the MC. An interpretation of this statement in the context of a factorization framework for hadron colliders is still lacking. Reference [50] advocates extracting directly the top $\overline{\mathrm{MS}}$ mass from the top pair production cross section. The improvement at the current level of precision $\delta m_{t} \leq 1 \mathrm{GeV}$, however, is small [12] (see also the discussion of renormalon ambiguity, above). The extracted top $\overline{\mathrm{MS}}$ mass might be affected by the findings reported in Ref. [15].

7. Higher-order corrections Missing higher-order corrections can be an important source of uncertainty in the determination of the top mass. These are typically added through NLO calculations $[38,40,51,52]$ and for the case of the total cross section through approximate NNLO calculations $[12,46,50,53]$ (for calculations in full NNLO, see the discussion in Sect. 3.3). A particularly sensitive issue is the matching of NLO top-quark calculations to parton showers; see [54-56].

8. Non-perturbative corrections Non-perturbative corrections mostly affect the MC modeling of the final state. These include hadronization, in particular of the finalstate partons that inherit the top quark color charges (which causes an unavoidable non-perturbative exchange of energy with the rest of the event), hadron and $\tau$ decays (including the $B$ hadron decays), underlying event, and possible additional non-perturbative phenomena such as color reconnections or other collective phenomena. Depending on how the corrections to the cross sections in Eq. 1 are performed, these uncertainties enter either on the experimental or theoretical side of the equation. The underlying-event, hadronization, and particle-decay corrections are typically split into two components; firstly, a process-independent jet-energy-scale (JES) systematic, determined mainly from photon+jet and dijet events and applied to the jets in top events, and secondly a dedicated top-specific systematic obtained by varying the hadronization and underlying-event modeling on $t \bar{t} \mathrm{MC}$ samples and evaluating the change in the extracted topquark mass. Since there is currently no systematic way of treating the correlation between these two components, double counting between them is a significant concern, with different experiments taking different approaches. This is an area on which further input from the MC modeling community could be beneficial. A study of colorreconnection effects in the special case of $e^{+} e^{-}$collisions found very small effects $<100 \mathrm{MeV}$ [57], but toy models show that the effect in hadron collisions may be as large as $0.5 \mathrm{GeV}$ [58]. More physical models and better constraints are required to reduce this uncertainty further, for instance by allowing one to bound it, rather than merely switching it on and off. Non-perturbative corrections can also be introduced through final-state interactions in the presence of strong jet vetoes [59]. Inclusive measurements like the methods described in Sects. 3.4 and 3.5 are likely to suffer least from such non-perturbative effects.

9. Contributions from physics beyond the standard model It is possible that some yet-undiscovered physics beyond the standard model (BSM) might influence the various measurements used to extract the top quark mass. Given that in the context of top mass extraction experimental measurements have so far always been compared with predictions based on the SM, the possibility arises that there might be a bias in the determination of the top quark mass due to new physics. While it is unlikely that such new physics can cause large corrections, ${ }^{1} \mathcal{O}(1 \mathrm{GeV})$ modifications to $m_{t}$ cannot be excluded at present. A first dedicated study of BSM contributions to $m_{t}$ determination is ongoing [60]. Application to top mass measurements of the work reported in Refs. [61,62] may also be useful for disentangling BSM contributions (although this will likely require the inclusion of NLO QCD corrections).

\section{Top mass determination at hadron colliders}

A major collection of experimental methods is available in [63]. Here we highlight a few that have already proven useful or appear to be promising:

\footnotetext{
${ }^{1}$ For example, the CMS endpoint top mass determination (see Sect. 3.2) is based on kinematical considerations, i.e. it has reduced sensitivity to the top quark production mechanism.
} 
1. Matrix element methods The most precise measurements of $m_{t}$ from the Tevatron use the matrix element method $[64,65]$, in which the measured objects are compared with expectations from the LO $t \bar{t}$ production and decay diagrams convoluted with the detector response. The method derives much of its power from the fact that the likelihood for each event to be consistent with both $t \bar{t}$ and background production is calculated; greater weight is assigned to events that are more likely to be from $t \bar{t}$ when measuring $m_{t}$. In addition, the hadronically decaying $W$ boson in $\ell+$ jets events provides an in situ constraint on the jet response, substantially reducing the systematic uncertainty. An NLO theory approach is currently being developed [66].

2. Ideogram and template methods The current generation of CMS analyses, which are among the most precise $m_{t}$ measurements, use ideogram techniques. The ideogram corresponding to the most probable solution for the mass is determined on an event-by-event basis. These are then summed over the full dataset to determine an "integrated ideogram". The top mass is then determined by fitting this to a Monte Carlo spectrum for the same number of events. The MC spectrum is determined as a function of $m_{t}$ (CMS — all jets) or $m_{t}$ and JES (CMS_lepton and jets). The dilepton channel is handled in a similar way using the analytical matrix weighting technique (AMWT) to treat the two-neutrino ambiguities. Regarding Monte Carlo generators, CMS uses Madrgraph [67] (LO ME generator) with Pythia [68,69] for the parton showering. The ATLAS Collaboration uses similar "template" methods. The main differences with respect to the CMS analyses are that the ATLAS Collaboration currently uses three parameters $\left(m_{t}\right.$, lightJES, bJES) for their lepton + jets analysis as well as POWHEG [70-72] + Pythia for event generation.

3. Extraction from the total cross section $\sigma_{\text {tot }}$ The total inclusive $t \bar{t}$ cross section at a given collider depends on $m_{t}$, so the measured cross section can be used to constrain $m_{t}$. Extractions of the top mass from $\sigma_{\text {tot }}$ have been performed in $[12,46,50,53,73]$ using NLO+NNLL or approximate NNLO cross section calculations. Very recently a first analysis performed in full NNLO+NNLL appeared [74]. The sensitivity of $\sigma_{\text {tot }}$ to the top mass is relatively low (few \%), so this method is not competitive in precision with other existing methods. On the other hand the method uses an observable based on a well-defined top mass, has small uncertainties due to perturbative and non-perturbative effects, and is not very sensitive to top width effects.

4. The $J / \psi$ method [75] In about one in $10^{5}$ top quark decays, the fragmentation products of the $b$ quark will include a $J / \psi$ decaying to $\mu^{+} \mu^{-}$. If the $W$ boson from the same top quark also decays leptonically, the three- lepton invariant mass is sensitive to $m_{t}$. The other top quark is only used to discriminate $t \bar{t}$ production from background. The strength of this method is that the main systematic uncertainties arise from different sources than in other methods (primarily $b$ fragmentation), and they may be smaller. Moreover, no $t \bar{t}$ reconstruction takes place i.e. the method is inclusive at any order in perturbation theory. These potential advantages must be weighed against the statistical limitations arising from requiring a $J / \psi$ candidate. MC studies of this method are reported in [76-78], and the uncertainty from $b$ fragmentation was studied at NLO in [79-81]. A NLO study, with factorized production and decay, was performed in Ref. [52]. The complete NLO result including production/decay interferences, off-shell effects and backgrounds, was computed in Refs. [38,39] (the $B$ mesons in this work are treated as $b$-jets). Additional error estimates, performed within this study, can be found in Sect. 3.4 below. A related measurement of the top mass, using the lepton and $b$-jet invariant mass, has been performed by the ATLAS Collaboration [82].

5. Dilepton-specific methods In the same spirit as the $J / \psi$ method, it may be advantageous to measure $m_{t}$ using kinematic properties (e.g. the invariant mass and $p_{T}$ ) of the lepton pair in dilepton $t \bar{t}$ candidates (selected as pair of leptons and possibly two $b$ 's, without requiring $t \bar{t}$ reconstruction) [83]. These observables should have a smaller sensitivity to the modeling of hadronic observables (showering and jets). Such measurements can be compared versus complete NLO calculations [38-40], as well as versus standard MC generators. This approach may not be as sensitive to the value of $m_{t}$ as other methods, but it offers very different systematics, and therefore may help to reduce the overall uncertainty on the world-average $m_{t}$. First measurements of top pair differential distributions in dilepton final states have already appeared [84]. See also the related discussion in Sect. 3.5 below.

In the near to medium term (i.e. prior to the construction of a lepton collider capable of performing a $t \bar{t}$ threshold scan), improvement in the precision with which we know $m_{t}$ will depend on:

- Extraction of the top mass with new methods that have alternative systematics (like 4 and 5 in Sect. 3). Such extractions will either validate the current precision in the available top mass measurements or highlight the need for additional scrutiny. Further phenomenological and experiment studies of these new methods are needed.

- Decreasing the perturbative uncertainty in currently used matrix element methods by applying future extension of the work in Ref. [66]. It remains an open question if top 
width effects and non-perturbative effects can also be reduced this way.

- Improved understanding of the relation between MC mass and standard quark masses, such as the pole mass. Work along these lines has been reported in [11]; see also Ref. [28, Appendix C].

In the following we review, and present estimates, for the capabilities of various methods for top mass determination. The methods can be split into "conventional" (Sect. 3.1), "other available" (Sects. 3.2, 3.3) or "under development" (Sects. 3.4, 3.5).

\section{1 "Conventional" top mass determination techniques}

As a model for the conventional collider mass measurements, we consider the CMS lepton-plus-jets [85], dilepton [86] and all-hadronic analyses [87]. These are currently the most precise measurements in each channel. The analyses use similar methods and result in measurements with comparable systematic uncertainties. To estimate the potential precision for the various $14 \mathrm{TeV}$ scenarios we have taken the CMS leptonplus-jet result $m_{t}=173.49 \pm 0.27$ (stat.) \pm 1.03 (syst.) $\mathrm{GeV}$ as representative and have performed extrapolations based on this. The results are presented in Table 1.

These are based on the 7 and $14 \mathrm{TeV}$ cross sections calculated using the full NNLO framework [88-91] with an allowance for a decreased trigger efficiency due to higher event rates and trigger thresholds. For the systematic errors, we assume that some of the soft QCD and fragmentation uncertainties will be constrained using the data from future LHC runs. We keep the initial- and final-state radiation and pdf uncertainties unchanged. Without a full simulation of the machine conditions, we are unable to model the effects of the increased merging of the top-decay products in moving to the higher energy. To allow for this and the uncertainties in the extrapolations we add in an additional $300 \mathrm{MeV}$ uncertainty to the mass measurement.

Table 1 Extrapolations based on the published CMS lepton-plus-jets analysis

\begin{tabular}{|c|c|c|c|c|c|c|}
\hline & Ref. [85] & Proje & tions & & & \\
\hline CM energy (TeV) & 7 & 14 & & & & \\
\hline Cross section (pb) & 167 & 951 & & & & \\
\hline Luminosity $\left(\mathrm{fb}^{-1}\right)$ & 5 & 100 & 100 & 300 & 300 & 3,000 \\
\hline Pileup & 9.3 & 19 & 30 & 19 & 30 & 95 \\
\hline Syst. $(\mathrm{GeV})$ & 0.95 & 0.7 & 0.7 & 0.6 & 0.6 & 0.6 \\
\hline Stat. $(\mathrm{GeV})$ & 0.43 & 0.04 & 0.04 & 0.03 & 0.03 & 0.01 \\
\hline Total & 1.04 & 0.7 & 0.7 & 0.6 & 0.6 & 0.6 \\
\hline Total (\%) & 0.6 & 0.4 & 0.4 & 0.3 & 0.3 & 0.3 \\
\hline
\end{tabular}

Table 2 Dominant systemic uncertainties for each scenario

\begin{tabular}{lc}
\hline Scenario & Dominant uncertainties \\
\hline Ref. [85] & Jet energy scale, hadronization, soft QCD, \\
& ISR/FSR \\
$100 \mathrm{fb}^{-1} / 19 \mathrm{PU}$ & Jet energy scale, hadronization, soft QCD, \\
& ISR/FSR \\
$100 \mathrm{fb}^{-1} / 30 \mathrm{PU}$ & Jet energy scale, hadronization, soft QCD, \\
& ISR/FSR, pileup \\
$300 \mathrm{fb}^{-1} / 19 \mathrm{PU}$ & Jet energy scale, hadronization, soft QCD, \\
$300 \mathrm{fb}^{-1} / 30 \mathrm{PU}$ & ISR/FSR \\
& Jet energy scale, hadronization, soft QCD, \\
$3,000 \mathrm{fb}^{-1} / 95 \mathrm{PU}$ & ISR/FSR, pileup \\
& Jet energy scale, hadronization, soft QCD, \\
& ISR/FSR, pileup \\
\hline
\end{tabular}

In Table 2 we summarize the dominant uncertainties for each scenario. While these are very similar, it should be noted that pileup and the associated uncertainties from the missing transverse energy and contamination of the underlying event are expected to become increasingly important as the collision energy and pileup are increased. We also note that the ISR/FSR uncertainly, which is one of the sub-leading uncertainties for [85] becomes one of the leading uncertainties for each of the 300 and $3,000 \mathrm{fb}^{-1}$ scenarios.

Based on the comparison of the results from [85] and the CMS combined result from the three channels shown at the TOP2012 Workshop [92], see also [93], we estimate that combinations of different channels for each of the $14 \mathrm{TeV}$ scenarios may lead to a small improvement in the projected precisions. We also note that the triggering on the all-hadronic events may prove difficult when running at very high luminosity and under high pileup conditions. This may prevent the effective use of this channel under these conditions.

\subsection{CMS endpoint method [94]}

This method is kinematical in nature and utilizes the correlation between the endpoints of the $M_{b \ell}$ and the $M_{\mathrm{T} 2 \mathrm{perp}}^{221}$ distributions and $m_{t}[95,96]$. It gives a mass measurement $m_{t}=173.90 \pm 0.90$ (stat.) ${ }_{-2.1}^{+1.70}$ (syst.) GeV. This was extrapolated using similar assumptions to that used for the CMS lepton-plus-jet method. A summary of the results is given in Table 3. As this technique is insensitive to pileup effects we only quote one extrapolation for each of the luminosity scenarios.

In Table 4 we summarize the dominant uncertainties for each scenario. As with the conventional analysis, these are fairly similar as a function of increasing luminosity. We also note that, unlike the conventional method, the ISR/FSR and pileup terms do not seem to play a role in the precision of the measurements, even at high luminosity. 
Table 3 Extrapolations based on the published CMS Endpoint analysis

\begin{tabular}{lllll}
\hline & Ref. [94] & Projections & \\
\hline CM energy (TeV) & 7 & & 14 & \\
Cross section (pb) & 167 & & 951 & \\
Luminosity $\left(\mathrm{fb}^{-1}\right)$ & 5 & 100 & 300 & 3,000 \\
Syst. $(\mathrm{GeV})$ & 1.8 & 1.0 & 0.7 & 0.5 \\
Stat. $(\mathrm{GeV})$ & 0.90 & 0.10 & 0.05 & 0.02 \\
Total & 2.0 & 1.0 & 0.7 & 0.5 \\
Total $(\%)$ & 1.2 & 0.6 & 0.4 & 0.3 \\
\hline
\end{tabular}

Table 4 Dominant systemic uncertainties for each scenario

\begin{tabular}{ll}
\hline Scenario & Dominant uncertainties \\
\hline Ref. [94] & Jet energy scale, hadronization, soft QCD \\
$100 \mathrm{fb}^{-1}$ & Jet energy scale, hadronization, soft QCD \\
$300 \mathrm{fb}^{-1}$ & Jet energy scale, hadronization, soft QCD \\
$3,000 \mathrm{fb}^{-1}$ & Jet energy scale, hadronization \\
\hline
\end{tabular}

Although the terms listed in Tables 2 and 4 have a large overlap, we note that they are not $100 \%$ correlated so that combining the results from the two methods may be beneficial to the overall precision. This follows from the fact that, unlike the conventional analyses, the Endpoint method does not rely on Monte Carlo modeling to do an internal calibration. It is largely analytical with a data-driven model for the background.

We also note that the kinematical nature of this method makes it suitable to attempt top mass determination which is less likely to be affected by possible new physics contributions. Nonetheless, this important aspect of $m_{t}$ determination needs further study. Finally, one would like to study in more detail the effect of higher order corrections, for example, by comparing with the findings of Refs. [38,40].

\subsection{ATLAS three-dimensional template fit method [97]}

The ATLAS Collaboration has recently published a new determination of the top quark mass in the lepton+jets final state [97]. This analysis uses a three-dimensional template technique which determines the top quark mass together with a global jet energy scale factor (JSF), and a relative bquark jet to light-quark jet energy scale factor (bJSF), which allow important experimental systematic uncertainties to be reduced. The result is $m_{t}=172.31 \pm 0.23$ (stat) \pm 0.27 (JSF) \pm 0.67 (bJSF) \pm 1.35 (syst) GeV. The uncertainties labeled JSF and bJSF correspond to the statistical uncertainty of the global jet energy scale factor (JSF) and the relative b-jet to light-jet energy scale factor (bJSF). The in-situ determination of these global jet energy scale factors in the
3D fit has allowed the two dominant systematic uncertainties to be transformed into statistical uncertainties to a large extent. The residual jet energy scale uncertainty is combined together with a large number of other sources of uncertainty into "syst". The modeling of top quark production and decay has a non-negligible contribution.

\subsection{Top mass determination from $J / \Psi$ final states [75]}

Our estimate of the theory error is based on the NLO QCD calculation of Ref. [52] performed for LHC $14 \mathrm{TeV}$. The estimation of the statistical uncertainty is based on preliminary studies by the CMS Collaboration. Calculations for LHC $33 \mathrm{TeV}$ in leading order QCD are also available. ${ }^{2}$ From these results we conclude that $\left\langle M_{B \ell}\right\rangle\left(m_{t}\right)$ is not sensitive to the collider energy, if the same cuts are used. More restrictive cuts for LHC $33 \mathrm{TeV}$ lead to slight modification of the $\left\langle M_{B \ell}\right\rangle\left(m_{t}\right)$ dependence, but the theoretical error of the extracted $m_{t}$ remains largely unchanged. The main sources of theoretical error in the $J / \Psi$ method are scale variation and $B$-fragmentation. Modeling of $\left\langle M_{B \ell}\right\rangle$ in NNLO QCD could become possible during the $\mathrm{LHC} 13 \mathrm{TeV}$ run, which would reduce the scale variation by a factor of 2.5 . We estimate this possible improvement by comparing in Table 5 the scale and pdf uncertainty of the total inclusive cross section for LHC 13 and $33 \mathrm{TeV}$ at NLO and NNLO [88-91].

We use $m=173.3 \mathrm{GeV}$ with MSTW2008 [98] (with 68cl) and NNPDF2.3 [99] (with $\alpha_{s}\left(M_{Z}\right)=0.118$ and $n_{f}=5$ ) NLO and NNLO pdf sets.

The long-term limiting factor would be the uncertainty in $B$-fragmentation. As a benchmark, we take the DELPHI measurement [100] of the first moment of the fragmentation function $\langle x\rangle=0.7153 \pm 0.0052$, which has an uncertainty of about $0.7 \%$ (completely dominated by systematics). Such error in $\left\langle M_{B \ell}\right\rangle$ implies $\delta m_{t} \approx 0.9 \mathrm{GeV}$. A future dedicated ILC run at the $Z$-pole should be able to significantly improve the existing measurements of $B$-fragmentation. Such a measurement is likely to occur only after the end of the currently foreseen LHC operations but before the dedicated top threshold scan during the later phases of the ILC where, for the first time, measurement of a short-distance top quark mass with very high precision $\mathcal{O}(100 \mathrm{MeV})$ will be performed (see Sects. 4, 4.1, and 4.4).

The estimates for the total error are given in Table 6. The theoretical error is estimated as follows: for LHC 8 and $14 \mathrm{TeV}$ and luminosity up to $300 \mathrm{fb}^{-1}$ we take the error as estimated in Ref. [52]. For 3,000 $\mathrm{fb}^{-1}$ at $14 \mathrm{TeV}$ we assume that NNLO calculation will be available, which will decrease the scale uncertainty by a factor of 2.5 . At this point the dominant uncertainty is the one from $B$-fragmentation. For LHC

\footnotetext{
${ }^{2}$ We thank the authors of Ref. [52] for providing us with these additional estimates.
} 
Table 5 Scale and pdf uncertainty for the total inclusive $t \bar{t}$ cross section at 13 and $33 \mathrm{TeV}$

\begin{tabular}{|c|c|c|c|c|c|c|c|c|}
\hline & \multicolumn{4}{|c|}{ LHC $13 \mathrm{TeV}$} & \multicolumn{4}{|c|}{ LHC $33 \mathrm{TeV}$} \\
\hline & \multicolumn{2}{|c|}{$\delta_{\text {scale }}(\%)$} & \multicolumn{2}{|l|}{$\delta_{\text {pdf }}(\%)$} & \multicolumn{2}{|c|}{$\delta_{\text {scale }}(\%)$} & \multicolumn{2}{|l|}{$\delta_{\text {pdf }}(\%)$} \\
\hline & MSTW & NNPDF & MSTW & NNPDF & MSTW & NNPDF & MSTW & NNPDF \\
\hline NLO & $\begin{array}{l}+12.1 \\
-12.1\end{array}$ & $\begin{array}{l}+11.8 \\
-11.9\end{array}$ & $\begin{array}{l}+1.9 \\
-2.3\end{array}$ & $\begin{array}{l}+1.8 \\
-1.8\end{array}$ & $\begin{array}{l}+11.5 \\
-10.3\end{array}$ & $\begin{array}{l}+11.2 \\
-10.0\end{array}$ & $\begin{array}{l}+1.2 \\
-1.5\end{array}$ & $\begin{array}{l}+1.0 \\
-1.0\end{array}$ \\
\hline NNLO & $\begin{array}{l}+3.4 \\
-5.6\end{array}$ & $\begin{array}{l}+3.5 \\
-5.7\end{array}$ & $\begin{array}{l}+1.8 \\
-2.0\end{array}$ & $\begin{array}{l}+1.8 \\
-1.8\end{array}$ & $\begin{array}{l}+3.1 \\
-4.7\end{array}$ & $\begin{array}{l}+3.1 \\
-4.7\end{array}$ & $\begin{array}{l}+1.0 \\
-1.4\end{array}$ & $\begin{array}{l}+1.0 \\
-1.0\end{array}$ \\
\hline
\end{tabular}

Table 6 Extrapolations based on the $J / \Psi$ method

\begin{tabular}{|c|c|c|c|c|c|c|}
\hline & Ref. analysis & Proje & ections & & & \\
\hline CM energy $(\mathrm{TeV})$ & 8 & & 14 & & 33 & 100 \\
\hline Cross section (pb) & 240 & & 951 & & 5,522 & 25,562 \\
\hline Luminosity $\left(\mathrm{fb}^{-1}\right)$ & 20 & 100 & 300 & 3,000 & 3,000 & 3,000 \\
\hline Theory (GeV) & - & 1.5 & 1.5 & 1.0 & 1.0 & 0.6 \\
\hline Stat. (GeV) & 7.00 & 1.8 & 1.0 & 0.3 & 0.1 & 0.1 \\
\hline Total & - & 2.3 & 1.8 & 1.1 & 1.0 & 0.6 \\
\hline Total (\%) & - & 1.3 & 1.0 & 0.6 & 0.6 & 0.4 \\
\hline
\end{tabular}

at $100 \mathrm{TeV}$ we assume that the $B$-fragmentation uncertainty is reduced by a factor of 2 with the help of a dedicated future lepton collider measurement.

\subsection{Top mass determination from kinematic distributions}

The top quark mass can be extracted from $\sigma_{\text {tot }}$. The advantage of this method is that a mass is obtained in a rigorously defined mass scheme. Typically the extracted mass would be the top pole mass since existing higher-order calculations are (naturally) performed in that scheme. It is possible to convert such calculations into a short-distance mass scheme as was done in Refs. [12,50] for the $\overline{\mathrm{MS}}$ and $1 S$ [9] masses (for more on this point see also the discussion on Alternative top mass definitions in Sect. 2). The D0 experiment has attempted this approach [73]. Preliminary results have been presented by both the ATLAS and the CMS Collaborations. The uncertainty on the extracted top quark mass amounts to approximately $3 \%$. Although the recently derived NNLO result [ 88 91] has not yet been fully utilized in this regard (however, see Ref. [74]), significant future improvements within this approach are unlikely given that the uncertainty in $\sigma_{\text {tot }}$ at present arises from a number of competing sources [101]. Ultimately the potential of this method is expected to be limited by the relatively small sensitivity of the cross section with respect to the top quark mass.

Kinematic differential distributions offer improved sensitivity to $m_{t}$ and several methods for the extraction of the top pole mass have been studied.
Reference [102] suggested $m_{t}$ extraction from the invariant mass distribution of $t \bar{t}$ pairs produced in events in association with a hard jet. The sensitivity is improved well beyond what can be achieved with the total cross section. The authors claim that uncertainties related to uncalculated higher-order corrections or uncertainties in the parton distribution functions are expected to affect the mass measurement by less than $1 \mathrm{GeV}$. The impact of top decays and experimental uncertainties - evaluated in a generic detector simulationis also expected to be sub-GeV.

The extraction of $m_{t}$ from leptonic kinematic distributions in dilepton events [83] is less affected by MC modeling and non-perturbative corrections, thus reducing an important source of uncertainty in the current top mass extractions. The only currently available study of $m_{t}$ extraction from dilepton events has been performed for LHC $14 \mathrm{TeV}$ in Ref. [52] where the authors find the possibility for extracting $m_{t}$ with precision of about $1.5 \mathrm{GeV}$. Such a precision is similar to the one from the $J / \Psi$ method. Further exploration of the systematics in this method is needed and studies are currently under way [83].

\section{Top mass determination at lepton colliders}

Current theoretical understanding of top quark threshold production at lepton colliders suggests (see Sect. 4.1 below) that it is feasible to determine a short-distance top quark mass with a precision of about $100 \mathrm{MeV}$, the top quark width with a precision of about $40 \mathrm{MeV}$ and the top quark Yukawa coupling with a precision of about $50 \%$. Such a precision is substantially higher than the ultimate precision expected at hadron colliders.

Several proposals for lepton colliders-mainly linear $e^{+} e^{-}$colliders - have been put forward so far. The International Linear Collider (ILC [103]) is a $e^{+} e^{-}$machine based on superconducting radio-frequency cavities. The Compact Linear Collider (CLIC [104]) has drive beam scheme capable of operating at multi-TeV energies. Both ILC and CLIC are expect to collect $100 \mathrm{fb}^{-1}$ after only few months of operation. A circular $e^{+} e^{-}$collider with a circumference of approximately $80-100 \mathrm{~km}$ could also reach the $t \bar{t}$ production thresh- 


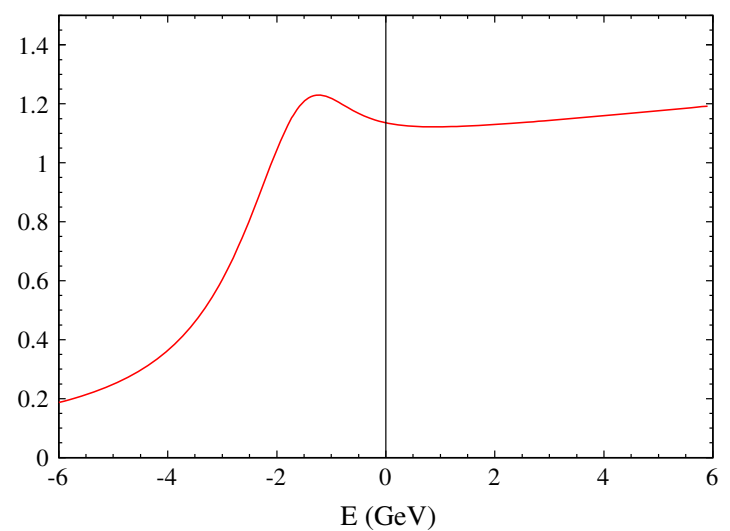

(a)

Fig. 1 a Typical threshold behavior of the (normalized) total $e^{+} e^{-} \rightarrow$ $t \bar{t}$ cross section with Coulomb and finite-width effects taken into account, $E=\sqrt{s-4 m_{t}^{2}}$. b Realistic simulations of various observ-

old (TLEP [105]). Research and development toward a muon collider is also ongoing [106].

The most promising method for high-precision extraction of the top quark mass is through a scan of the $t \bar{t}$ production threshold [107]. The authors of Ref. [108] find that a fourparameter fit including the top quark mass and width, the strong coupling constant and the top Yukawa coupling can yield a statistical precision of several tens of $\mathrm{MeV}$ on the top quark mass. Calculations of the production cross section in the threshold region $[35,109,110]$ have since reached a precision of few percent. The potentials of ILC and CLIC have been revisited [111] with realistic luminosity spectra for both machines, a detailed simulation of the detector response and an evaluation of the dominant systematic uncertainties. Assuming total integrated luminosity of $100 \mathrm{fb}^{-1}$, statistical uncertainty of $34 \mathrm{MeV}$ on the (1S) top quark mass when extracted from a 10-step threshold scan was found there.

Top quark mass measurements can also be performed at center-of-mass energies away from threshold. Above threshold (i.e. for $\sqrt{s}>2 m_{t}$ ) the top mass extracted from the invariant mass distribution of the reconstructed top quark decay products has excellent statistical precision; Ref. [111] quotes statistical uncertainty of $80 \mathrm{MeV}$ combining the events collected in the semi-leptonic and fully hadronic decay channels for $100 \mathrm{fb}^{-1}$ at $\sqrt{s}=500 \mathrm{GeV}$.

The rate for single top production $\left(e^{+} e^{-} \rightarrow t \bar{b} W^{-}\right.$and the charge conjugate process) depends strongly on the top quark mass for $\sqrt{s}<2 m_{t}$. The cross section for this process

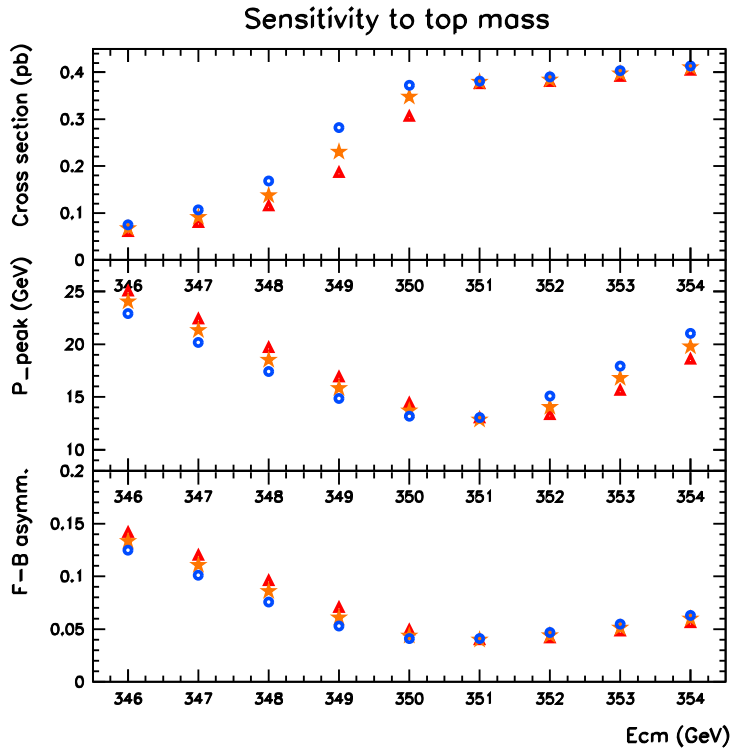

(b)

ables for the $t \bar{t}$ threshold production in $e^{+} e^{-}$collisions with the beam effects taken account [108]. Sensitivity to the top quark mass is indicated with the different symbols denoting $200 \mathrm{MeV}$ steps in top mass

is very small (less than a femtobarn for $\sqrt{s}$ below $300 \mathrm{GeV}$ ). Given the likely prospect that a future ILC will be operating for several years at energy around $250 \mathrm{GeV}$ before any top threshold measurement can be done, an exhaustive study of the possibilities for top mass determination below threshold is highly desirable.

\subsection{Theory of $t \bar{t}$ production near threshold at $e^{+} e^{-}$ colliders}

The dynamics of top pair production at threshold is controlled mainly by two opposing effects. Firstly, due to the strong interactions, the non-relativistic quark-antiquark pair tends to form a series of Coulomb-like bound states below threshold ("toponium"). Secondly, due to the weak interactions, the large decay width of the top quark (which is comparable to its Coulomb binding energy) smears out the sharp would-be resonances in the cross section. The interplay of these two effects leaves a single well-pronounced peak at $\sqrt{s_{\text {res }}} \approx 2 m_{t}$, which roughly corresponds to the would-be toponium ground state (see Fig. 1a).

The expression for the resonance cross section, $\sigma_{\text {res }} \sim$ $\alpha_{s}^{3} /\left(m_{t} \Gamma_{t}\right)$, reveals strong dependence on the top quark mass and width as well as on the strong coupling constant. Since $\Gamma_{t} \gg \Lambda_{\mathrm{QCD}}$, the top quark decays well before it hadronizes, i.e. the top quark width serves as an infrared cutoff which makes the process perturbative in the whole threshold region [19-21]. With non-perturbative effects fully under control, 


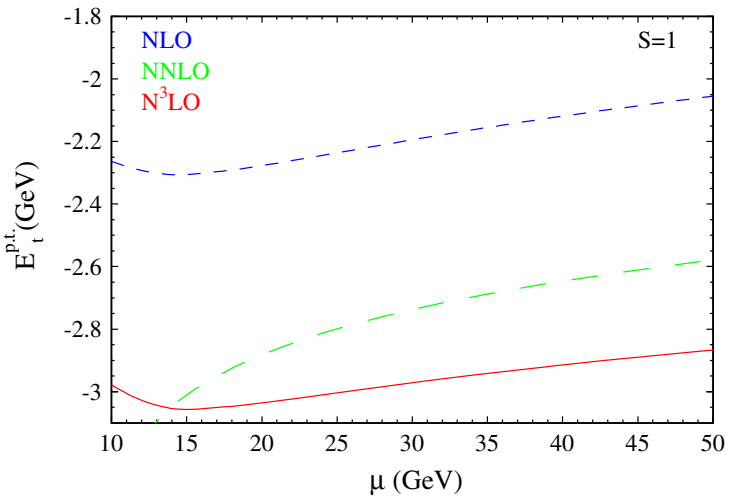

(a)

Fig. 2 Structure of perturbative expansion for a the resonance energy counted from the threshold [115] and $\mathbf{b}$ the (normalized) resonance cross section [128] in $e^{+} e^{-} \rightarrow t \bar{t}$. Subsequent approximations are plotted as

perturbative QCD gives a reliable theoretical description of the $t \bar{t}$ threshold production.

The accuracy of the approximation for $\sigma_{\text {res }}$ is limited mainly by its convergence, i.e. by the number of known terms in its perturbative expansion. Systematic calculation of the higher-order corrections in heavy quarkonium systems is based on the non-relativistic effective theory of (potential) NRQCD [112-114] which involves simultaneous expansions in the strong coupling constant and in the heavy quark velocity. The perturbative analysis has been pushed up to the NNLO by several groups [26]. The NNLO corrections to the cross section turned out to be huge despite the renormalization group suppression of the strong coupling at the characteristic mass scales.

A few conjectures have been made relating the slow convergence of the perturbation theory to the infrared renormalon contribution to the top quark pole mass, and to the corrections enhanced by powers of the logarithms of the heavy quark velocity in the case of the cross section. Estimates of the missing higher-order corrections have been done based on these assumptions. In particular, the use of various "threshold" or "short-distance" mass parameters free of infrared renormalon have been suggested in order to improve the convergence of the series for the resonance energy [810]. As it turns out, however, complete control over the $\mathrm{N}^{3} \mathrm{LO}$ corrections is ultimately necessary for a rigorous quantitative analysis of threshold production. Significant progress has been achieved in this field [115-127] and the main results are reviewed below.

\subsection{Resonance energy and top quark mass determination}

The total $\mathcal{O}\left(\alpha_{s}^{3}\right)$ correction to the leading order toponium ground-state energy has been obtained in [115]. The renor-

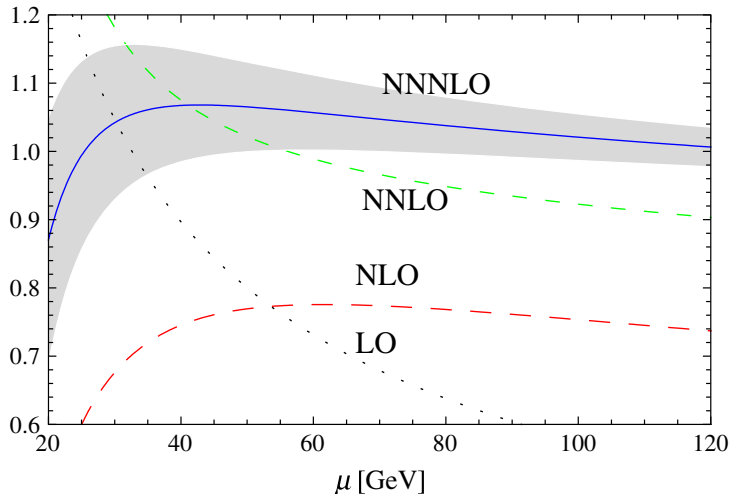

(b)

functions of the strong coupling normalization scale. The shaded area represent the uncertainty due to yet unknown three-loop Wilson coefficient

malon, logarithmic, and "generic" third order contributions turn out to be comparable in magnitude with no particular contribution saturating the total result. As shown in Fig. 2a, the third order correction stabilizes the series in the pole mass scheme and considerably reduces the scale dependence of the NNLO approximation. A crucial feature of the $\mathrm{N}^{3} \mathrm{LO}$ approximation is that it already contains all the effects characteristic to the threshold dynamics including the relativistic and ultrasoft (retardation) corrections. No qualitatively new effects exist beyond the third order of perturbation theory and the higher-order corrections are expected to be under control.

The numerical analysis of Ref. [115] produces a simple relation between the resonance energy and the top quark pole mass

$\sqrt{s_{\mathrm{res}}}=\left[1.9833+0.007 \frac{m_{t}-174.3 \mathrm{GeV}}{174.3 \mathrm{GeV}} \pm 0.0009\right] \times m_{t}$,

including the effect from the finite top quark width and the uncertainties in $\alpha_{s}\left(M_{Z}\right)=0.118 \pm 0.003$ and from unknown high-order terms. This corresponds to a theoretical uncertainty of about $80 \mathrm{MeV}$ in the extracted pole mass. Though the asymptotic character of the series for the binding energy in the pole mass scheme is not manifest through the third order (cf. Fig. 2a), the factorial divergence of the perturbative coefficients is expected in higher orders due to the infrared renormalon contribution, and the quoted uncertainty should be attributed to the fixed (third) order perturbative determination of the pole mass. The use of a less infrared sensitive mass parameter removes this ambiguity and apparently further reduces the error; for example, an uncertainty of $40 \mathrm{MeV}$ in the determination of the "conventional" shortdistance $\overline{\mathrm{MS}}$ mass $\bar{m}_{t}\left(\bar{m}_{t}\right)$ is quoted in [129]. Note that the series for the $\overline{\mathrm{MS}}$ mass converges for the strong coupling con- 
stant renormalized at $\mu \sim m_{t}$ as one would expect from a short-distance quantity, while the series for the infrared sensitive pole mass appears convergent at the soft renormalization scale $\mu \sim \alpha_{s} m_{t}$. However, the $\mathrm{N}^{3} \mathrm{LO}$ analysis requires the $\mathcal{O}\left(\alpha_{s}^{4}\right)$ perturbative relation between the pole and the $\overline{\mathrm{MS}}$ mass (currently known to $\mathcal{O}\left(\alpha_{s}^{3}\right)[13,14]$ ) and one has to rely on an assumption about the structure of the corresponding perturbative series [130], which may introduce an additional uncertainty. The calculation of the four-loop mass relation is, therefore, crucial for the determination of the short-distance mass with such an accuracy. At the same time the pole mass is a more natural parameter for the description of the invariant mass distribution of the top quark decay product as compared to the $\overline{\mathrm{MS}}$ or the threshold masses. The comparison of the values extracted from the invariant mass distribution and from the threshold energy scan may give a realistic estimate of the experimental and theoretical uncertainties.

\subsection{Threshold cross section}

The evaluation of the threshold cross section through $\mathrm{N}^{3} \mathrm{LO}$ is one of the most challenging problems of perturbative QCD. Currently the bulk of the third order corrections to the threshold cross section is available [116,118-125] with only a few Wilson coefficients still missing. The analysis is likely to be completed in the nearest future.

The structure of the perturbative series for the cross section is shown in Fig. 2b. As in the case of the resonance energy, the third order correction stabilizes the series and the accuracy of the $\mathrm{N}^{3} \mathrm{LO}$ approximation is likely to be about $3 \%$, or even better. Further refinement of the perturbative analysis may be achieved by resummation of the higher-order logarithmically enhanced corrections through effective theory renormalization group methods [131-134]. At this level of accuracy the electroweak effects become important. A consistent treatment of the top quark finite lifetime beyond the resonance approximation has been obtained through $\mathrm{N}^{2} \mathrm{LO}[36,37]$. The one-loop electroweak corrections to the cross section have been considered in $[135,136]$. Besides the total cross section, differential observables including forward-backward asymmetry and the top quark momentum distribution are known through NNLO up to non-factorizable effects in the top quark finite lifetime [9, 137].

\subsection{Threshold $t \bar{t}$ production at $e^{+} e^{-}$colliders: experimental simulations}

Realistic simulations of the $t \bar{t}$ threshold production have been performed in [108]. This study assumes a 9-point energy scan around the $t \bar{t}$ threshold where the nominal center-of-mass energy is varied between 346 and $354 \mathrm{GeV}$, in $1 \mathrm{GeV}$ steps, with an additional energy point taken well below threshold to measure the background. The assumed integrated luminosity per energy point is $30 \mathrm{fb}^{-1}$, for a total of $300 \mathrm{fb}^{-1}$ used in the full scan. This simulation takes into account the experimental uncertainties related to the detector effects, event selection efficiency, and the statistics, as well as an estimated theoretical uncertainty of $3 \%$ in the normalization of the cross section.

At each energy point, three observables are considered: the total cross section, the peak of the top quark momentum distribution, and the forward-backward asymmetry. The simulations show the total cross section to have an estimated experimental error of about $3 \%$, much below the one of the differential observables. No theoretical uncertainties on the differential observables have been taken into account yet. The results of the simulated scan for these three observables are shown in Fig. 1b.

As can be appreciated, the beam energy spread, bremsstrahlung, and beamstrahlung significantly smear the measured cross section and the precise determination of the (machine-dependent) luminosity spectrum is crucial for the reconstruction of the actual energy dependence of the cross section from the threshold scan. A multi-parameter fit including the top quark $1 \mathrm{~S}$ mass, top quark width and top quark Yukawa coupling is performed considering simultaneously the three observables mentioned above. The strong coupling constant $\alpha_{S}\left(M_{Z}\right)$ is used as an input value with an assumed uncertainty of \pm 0.001 . The resulting uncertainties on the top quark mass and width are 31 and $34 \mathrm{MeV}$, respectively. Note that these estimates do not account for any uncertainties on the nominal beam energy or the luminosity spectrum, which must be accurately known [138].

More recent studies have evaluated the potential precision on the top quark mass considering realistic luminosity spectra generated with the GUINEAPIG [139] program. In particular, Ref. [140] reports a detailed evaluation of the sensitivity of the top quark mass measurement to the ILC accelerator parameters. The nominal ILC parameters (Nominal) are compared to two alternative machine parameter known as $\operatorname{Low} Q$ and $L o w P$, that have reduced and increased beamstrahlung, respectively. Reference [111] has compared the top quark mass extraction form the threshold scan using luminosity spectra of the (nominal) ILC and CLIC, where beamstrahlung plays a more important role.

As an example, Fig. 3 shows the bare $t \bar{t}$ threshold as a function of center-of-mass energy near threshold, as well as the effective cross sections after convolution with the total luminosity spectrum, for the Nominal, Low $Q$ and LowP ILC machine parameters. The effective luminosity of the machine is clearly reduced due to the combined effects of bremsstrahlung, beamstrahlung and energy spread. The impact on the sensitivity is rather small: the statistical uncertainty on the top quark mass extracted at CLIC, with a very substantial increase in the level of beamstrahlung level, is 


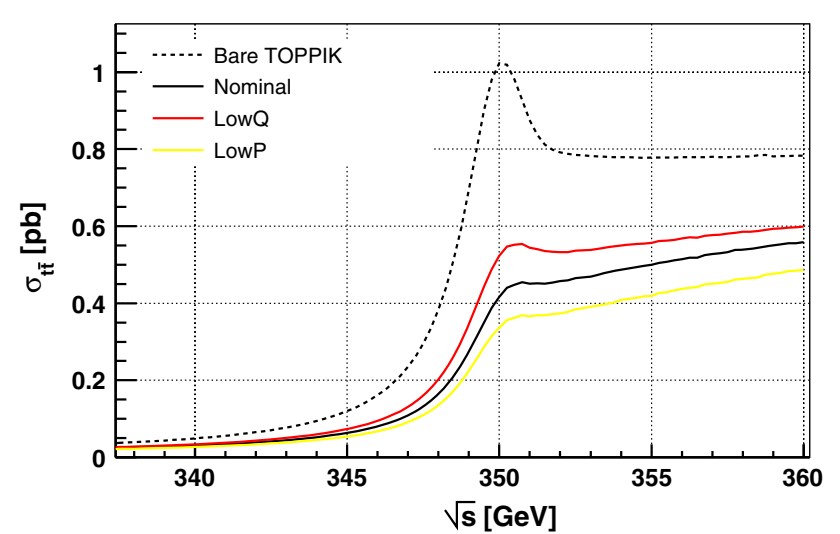

Fig. 3 Top quark pair production cross section in $e^{+} e^{-}$scattering near the $t \bar{t}$ threshold. The NNLO prediction based on the TOPPIK program [9], not including beam effects, is shown as the dashed line. Also shown are the predicted cross sections after convolution of the beam effects (beam energy spread, bremsstrahlung and beamstrahlung) corresponding to three different sets of ILC accelerator parameters (see text for details)

degraded by a few $\mathrm{MeV}$ with respect to the ILC [111]. An accurate knowledge of the effect on the shape of the cross section in the threshold region is, however, required to avoid a large systematic contribution to the extracted mass. While bremsstrahlung can be accurately predicted, the impact of beamstrahlung and beam energy spread (a much smaller contribution to the luminosity spectrum) must be determined experimentally. A detailed study [141] has been performed on how to reconstruct the luminosity spectrum from Bhabha events measured with the tracking detectors and calorimeters, taking into all relevant theoretical and experimental effects. This study shows that, in the context of the CLIC accelerator at $\sqrt{s}=3 \mathrm{TeV}$, the luminosity spectrum can be reconstructed to better than $5 \%$ between the nominal and about half the nominal center-of-mass energy. Pending a precise estimate of the resulting systematic uncertainty on the top quark mass measurement, a conservative $50 \mathrm{MeV}$ uncertainty based on early studies is assumed here.

The uncertainty on the nominal beam energy contributes a further systematic uncertainty. Recent studies in the context of the ILC [142,143] suggest that beam energy resolutions of $10^{-4}$ should be readily achievable. Therefore, the uncertainty in $\sqrt{s_{\text {res }}} / 2$ induced from the beam energy measurement is assumed to be $35 \mathrm{MeV}$ and independent of luminosity and machine parameter sets.

In summary, for a $300 \mathrm{fb}^{-1}$ threshold scan, the total expected uncertainty on a short-distance top quark mass is $\sim 100 \mathrm{MeV}$, resulting from the sum in quadrature of the following contributions: a statistical uncertainty of order $30 \mathrm{MeV}$ (from Ref. [108], confirmed to be possible also with $100 \mathrm{fb}^{-1}$ from a two-parameter fit in a recent study in Ref. [111]), $35 \mathrm{MeV}$ (beam energy), $50 \mathrm{MeV}$ (luminosity spectrum) and $80 \mathrm{MeV}$ (from the conversion of $s_{\text {res }}$ into $m_{t}$ according Eq. 3). Given the dominance of systematic uncertainties, it should be possible to reduce the integrated luminosity used in the threshold scan without significantly degrading the total uncertainty.

\subsection{Top quark mass from a reconstruction of the top-decay} products

At an $e^{+} e^{-}$collider the top quark mass can also be measured via reconstruction in the continuum, following approaches similar to those being pursued at the Tevatron and the LHC. One could a priori hope that the cleaner environment at an $e^{+} e^{-}$collider would allow smaller systematic uncertainties and thus improve upon the measurements from hadron colliders.

Full simulation studies on the top quark mass via direct reconstruction at an $e^{+} e^{-}$collider have been carried out in both the fully hadronic $\left(e^{+} e^{-} \rightarrow t \bar{t} \rightarrow q \bar{q} b q \bar{q} b\right)$ and semileptonic $\left(e^{+} e^{-} \rightarrow t \bar{t} \rightarrow \ell \nu b q \bar{q} b\right)$ decay channels [144146]. These studies have shown that statistical uncertainties on the top quark mass below $100 \mathrm{MeV}$ per decay channel are possible assuming an integrated luminosity of $100 \mathrm{fb}^{-1}$ at $\sqrt{s}=500 \mathrm{GeV}$. A similar statistical uncertainty is obtained for the measurement of the top width.

Similarly to the case of hadron colliders, systematic uncertainties are again expected to be the limiting factor. At present only limited information on the anticipated experimental and theoretical systematic uncertainties at an $e^{+} e^{-}$collider exists. Nevertheless, it is possible to obtain a rough lower limit on the total systematic uncertainty. The expected uncertainty due to fragmentation/hadronization modeling is $\sim 250$ (400) $\mathrm{MeV}$ in case of the semi-leptonic (fully hadronic) decay channel [147]. Reconnection effects in the final state could contribute uncertainties at the level of few hundred MeV. Preliminary studies suggest that Bose-Einstein correlations could contribute an uncertainty of $\sim 100-250 \mathrm{MeV}$ [147], while color-reconnection effects could also lead to an uncertainty of $\mathcal{O}(100) \mathrm{MeV}$ [148]. Finally, there is a theoretical uncertainty in the relation between the maximum of the invariant mass distribution and the mass parameter in the QCD Lagrangian.

It would be desirable to update these estimates taking advantage of the most recent developments in both event generators and experimental techniques for in situ constraining systematic uncertainties at hadron colliders. Taking into account all these contributions, and the fact that we have not considered experimental systematic uncertainties (e.g. jet energy calibration), it is difficult to imagine that the total systematic uncertainty would be less than $\left(\Delta m_{t}\right)_{\text {syst }} \sim$ $500 \mathrm{MeV}$, completely dominating this measurement. Thus the threshold scan clearly beats the direct reconstruction of the top quark mass in precision. The latter, however, can be 
used for additional control of systematic uncertainty in the threshold measurements.

\section{Conclusions}

In the course of the 2013 Snowmass process, and during the preparation of this document, we have analyzed the theoretical and experimental aspects of the problem of top quark mass determination. We have reached the following conclusions that reflect the past developments and future prospects in this field:

- Need for precision in $m_{t}$ determination The current precision with which the top quark pole mass $m_{t}$ is known, $\delta m_{t} \leq 1 \mathrm{GeV}[6,7]$, is already impressive; indeed the EW precision tests [1] are currently limited by the uncertainty in $m_{W}$ rather than in $m_{t}$. Nonetheless, motivation for increased precision may come from cosmology $[4,5]$, more fundamental issues in particle physics $[2,3]$, or a discovery of beyond the Standard Model physics at the LHC. We estimate that some methods for top mass determination at the LHC might lead to top pole mass extraction with total uncertainty as low as 500$600 \mathrm{MeV}$. Delivering such precision at the LHC will, however, be challenging and it remains to be seen if it can be achieved in practice. In the meantime, the most pressing issue is the relationship between the top quark mass measured at hadron colliders and a well-defined quark mass. Meaningful improvement in the precision will therefore likely require the application of several current and novel experimental methods that are sensitive to different effects, and also advances in the theoretical understanding of the relationship between measured and fundamental quantities. A significant increase in precision, reaching $\delta m_{t} \leq 100 \mathrm{MeV}$ in a short-distance scheme, can be achieved at a future lepton collider.

- A comprehensive collection of $m_{t}$ determination techniques This paper contains a comprehensive collection of top mass extraction methods for hadron colliders. These are methods that have been used in the past, are in current use or are under development. We discuss the salient features of each method and present estimates for the precision reach for some of them.

- Recommendations for further studies Going beyond the methods discussed in this paper, we point to two problems that have not been studied so far and that we think will be playing an increasingly important role in the future.

1. The possibility of BSM "contamination" in the various top mass measurements [60]. Both modeldependent and model-independent studies would be very useful.
2. The most precise known method for extracting $m_{t}$ is from a threshold scan at a future lepton collider. At present, however, it appears that the most likely lepton collider to be built is an ILC with a first stage operating at c.m. energy significantly below the $t \bar{t}$ threshold. The current expectation is that such first stage will be operational for a number of years; moreover, its energy upgrade might be affected by future considerations (like funding, for example). For this reason it is important to fully explore the possibility for top mass extraction at below-threshold energies through, for example, single top production. Such studies are lacking at present.

Acknowledgments Paper written within the Snowmass Energy Frontier working group HE3: Fully Understanding the Top Quark. We would like to thank Giorgio Cortiana, Pedro Ruiz Femenia, Andre Sailer and Frank Simon for discussions. The work of S. Mantry is supported by the U.S. National Science Foundation under grant NSF-PHY- 0705682. The work of A. Mitov is supported by ERC grant 291377 "LHCtheory: Theoretical predictions and analyses of LHC physics: advancing the precision frontier".

Open Access This article is distributed under the terms of the Creative Commons Attribution License which permits any use, distribution, and reproduction in any medium, provided the original author(s) and the source are credited.

Funded by $\mathrm{SCOAP}^{3}$ / License Version CC BY 4.0.

\section{References}

1. M. Baak, M. Goebel, J. Haller, A. Hoecker, D. Kennedy, R. Kogler, K. Moenig, M. Schott et al., Eur. Phys. J. C 72, 2205 (2012). arXiv:1209.2716 [hep-ph]

2. G. Degrassi, S. Di Vita, J. Elias-Miro, J.R. Espinosa, G.F. Giudice, G. Isidori, A. Strumia, JHEP 1208, 098 (2012). arXiv:1205.6497 [hep-ph]

3. F. Bezrukov, M.Y. Kalmykov, B.A. Kniehl, M. Shaposhnikov, JHEP 1210, 140 (2012) arXiv:1205.2893 [hep-ph]

4. F.L. Bezrukov, M. Shaposhnikov, Phys. Lett. B 659, 703 (2008). arXiv:0710.3755 [hep-th]

5. A. De Simone, M.P. Hertzberg, F. Wilczek, Phys. Lett. B 678, 1 (2009). arXiv:0812.4946 [hep-ph]

6. T. Aaltonen et al., CDF and D0 Collaborations, Phys. Rev. D 86, 092003 (2012) arXiv: 1207.1069 [hep-ex]

7. ATLAS and CMS Collaborations, ATLAS-CONF-2012-095, CMS PAS TOP-12-001 (2012)

8. M. Beneke, Phys. Lett. B 434, 115 (1998). hep-ph/9804241

9. A.H. Hoang, T. Teubner, Phys. Rev. D 60, 114027 (1999). hep-ph/9904468

10. A. Pineda, JHEP 0106, 022 (2001). hep-ph/0105008.

11. A.H. Hoang, I.W. Stewart, Nucl. Phys. Proc. Suppl. 185, 220 (2008). arXiv:0808.0222 [hep-ph]

12. V. Ahrens, A. Ferroglia, M. Neubert, B.D. Pecjak, L.L. Yang, Phys. Lett. B 703, 135 (2011). arXiv:1105.5824 [hep-ph]

13. K.G. Chetyrkin, M. Steinhauser, Phys. Rev. Lett. 83, 4001 (1999). hep-ph/9907509

14. K. Melnikov, T. V. Ritbergen, Phys. Lett. B 482, 99 (2000). hep-ph/9912391 
15. F. Jegerlehner, M.Y. Kalmykov, B.A. Kniehl, Phys. Lett. B 722, 123 (2013). arXiv:1212.4319 [hep-ph]

16. S. Fleming, A.H. Hoang, S. Mantry, I.W. Stewart, Phys. Rev. D 77, 114003 (2008). arXiv:0711.2079 [hep-ph]

17. A.H. Hoang, A. Jain, I. Scimemi, I.W. Stewart, Phys. Rev. Lett. 101, 151602 (2008). arXiv:0803.4214 [hep-ph]

18. G.S. Bali, A. Pineda, Phys. Rev. D 69, 094001 (2004). hep-ph/0310130

19. V.S. Fadin, V.A. Khoze, JETP Lett. 46, 525 (1987) [Pisma Zh. Eksp. Teor. Fiz. 46, 417 (1987)]

20. M.J. Strassler, M.E. Peskin, Phys. Rev. D 43, 1500 (1991)

21. V.S. Fadin, V.A. Khoze, Sov. J. Nucl. Phys. 48, 309 (1988)

22. V.S. Fadin, V.A. Khoze, Sov. J. Nucl. Phys. 53, 692 (1991) [Yad. Fiz. 53, 1118 (1991)].

23. S. Fleming, A.H. Hoang, S. Mantry, I.W. Stewart, Phys. Rev. D 77, 074010 (2008). hep-ph/0703207

24. A. Jain, I. Scimemi, I.W. Stewart, Phys. Rev. D 77, 094008 (2008). arXiv:0801.0743 [hep-ph]

25. A.A. Penin, J.H. Piclum, PoS LL 2012, 067 (2012). arXiv:1209.3821 [hep-ph]

26. A.H. Hoang, M. Beneke, K. Melnikov, T. Nagano, A. Ota, A.A. Penin, A.A. Pivovarov, A. Signer et al., Eur. Phys. J. direct C 2, 1 (2000). hep-ph/0001286

27. S.V. Chekanov, V.L. Morgunov, Phys. Rev. D 67, 074011 (2003). hep-ex/0301014

28. A. Buckley, J. Butterworth, S. Gieseke, D. Grellscheid, S. Hoche, H. Hoeth, F. Krauss, L. Lonnblad et al., Phys. Rept. 504, 145 (2011). arXiv:1101.2599 [hep-ph]

29. G. Corcella, M.H. Seymour, Phys. Lett. B 442, 417 (1998). hep-ph/9809451

30. E. Norrbin, T. Sjöstrand, Nucl. Phys. B 603, 297 (2001). hep-ph/0010012

31. K. Hamilton, P. Richardson, JHEP 0702, 069 (2007). hep-ph/0612236

32. M.L. Mangano, M. Moretti, F. Piccinini, M. Treccani, JHEP 0701, 013 (2007). hep-ph/0611129

33. S. Schumann, F. Krauss, JHEP $\mathbf{0 8 0 3}, 038$ (2008). arXiv:0709.1027 [hep-ph]

34. J. Alwall, S. de Visscher, F. Maltoni, JHEP 0902, 017 (2009). arXiv:0810.5350 [hep-ph]

35. A.H. Hoang, C.J. Reisser, P. Ruiz-Femenia, Phys. Rev. D 82, 014005 (2010). arXiv:1002.3223 [hep-ph]

36. M. Beneke, B. Jantzen, P. Ruiz-Femenia, Nucl. Phys. B 840, 186 (2010). arXiv:1004.2188 [hep-ph]

37. A.A. Penin, J.H. Piclum, JHEP 1201, 034 (2012). arXiv: 1110.1970 [hep-ph]

38. A. Denner, S. Dittmaier, S. Kallweit, S. Pozzorini, JHEP 1210, 110 (2012). arXiv:1207.5018 [hep-ph]

39. A. Denner, S. Dittmaier, S. Kallweit, S. Pozzorini, Phys. Rev. Lett. 106, 052001 (2011). arXiv:1012.3975 [hep-ph]

40. G. Bevilacqua, M. Czakon, A. van Hameren, C.G. Papadopoulos, M. Worek, JHEP 1102, 083 (2011). arXiv:1012.4230 [hep-ph]

41. V.S. Fadin, V.A. Khoze, T. Sjostrand, Z. Phys. C 48, 613 (1990)

42. K. Hagiwara, Y. Sumino, H. Yokoya, Phys. Lett. B 666, 71 (2008). arXiv:0804.1014 [hep-ph]

43. Y. Kiyo, J.H. Kuhn, S. Moch, M. Steinhauser, P. Uwer, Eur. Phys. J. C 60, 375 (2009). arXiv:0812.0919 [hep-ph]

44. Y. Sumino, H. Yokoya, JHEP 1009, 034 (2010). arXiv:1007.0075 [hep-ph]

45. R. Bonciani, S. Catani, M.L. Mangano, P. Nason, Nucl. Phys. B 529, 424 (1998) [Erratum-ibid. B 803, 234 (2008)] hep-ph/9801375

46. M. Beneke, P. Falgari, S. Klein, C. Schwinn, Nucl. Phys. B 855, 695 (2012). arXiv:1109.1536 [hep-ph]

47. I.I.Y. Bigi, M.A. Shifman, N.G. Uraltsev, A.I. Vainshtein, Phys. Rev. D 50, 2234 (1994). hep-ph/9402360
48. M. Beneke, V.M. Braun, Nucl. Phys. B 426, 301 (1994). hep-ph/9402364

49. M.C. Smith, S.S. Willenbrock, Phys. Rev. Lett. 79, 3825 (1997). hep-ph/9612329

50. U. Langenfeld, S. Moch, P. Uwer, Phys. Rev. D 80, 054009 (2009). arXiv:0906.5273 [hep-ph]

51. K. Melnikov, M. Schulze, JHEP 0908, 049 (2009). arXiv:0907.3090 [hep-ph]

52. S. Biswas, K. Melnikov, M. Schulze, JHEP 1008, 048 (2010). arXiv: 1006.0910 [hep-ph]

53. M. Beneke, P. Falgari, S. Klein, J. Piclum, C. Schwinn, M. Ubiali, F. Yan, JHEP 1207, 194 (2012). arXiv:1206.2454 [hep-ph]

54. S. Frixione, P. Nason, B.R. Webber, JHEP 0308, 007 (2003). hep-ph/0305252

55. S. Frixione, P. Nason, G. Ridolfi, JHEP 0709, 126 (2007) arXiv:0707.3088 [hep-ph]

56. P. Nason, B. Webber, Ann. Rev. Nucl. Part. Sci. 62, 187 (2012). arXiv:1202.1251 [hep-ph]

57. V.A. Khoze, T. Sjöstrand, Phys. Lett. B 328, 466 (1994). hep-ph/9403394

58. P.Z. Skands, D. Wicke, Eur. Phys. J. C 52, 133 (2007) hep-ph/0703081 [HEP-PH]

59. A. Mitov, G. Sterman, Phys. Rev. D 86, 114038 (2012). arXiv:1209.5798 [hep-ph]

60. M. Czakon, A. Mitov, M. Papucci, J.T. Ruderman, A. Weiler, arXiv:1407.1043 [hep-ph]

61. K. Agashe, R. Franceschini, D. Kim, arXiv:1309.4776 [hep-ph]

62. K. Agashe, R. Franceschini, D. Kim, Phys. Rev. D 88, 057701 (2013). arXiv:1209.0772 [hep-ph]

63. A.B. Galtieri, F. Margaroli, I. Volobouev, Rept. Prog. Phys. 75, 056201 (2012). arXiv:1109.2163 [hep-ex]

64. V.M. Abazov et al., D0 Collaboration, Nature 429, 638 (2004)

65. J. Estrada, Ph.D. thesis, University of Rochester, FERMILABTHESIS-2001-07 (2001)

66. J.M. Campbell, W.T. Giele, C. Williams, JHEP 1211, 043 (2012) arXiv:1204.4424 [hep-ph]

67. J. Alwall, M. Herquet, F. Maltoni, O. Mattelaer, T. Stelzer, JHEP 1106, 128 (2011). arXiv:1106.0522 [hep-ph]

68. T. Sjostrand, S. Mrenna, P.Z. Skands, Comput. Phys. Commun. 178, 852 (2008). arXiv:0710.3820 [hep-ph]

69. T. Sjostrand, S. Mrenna, P.Z. Skands, JHEP 0605, 026 (2006) hep-ph/0603175

70. P. Nason, JHEP 0411, 040 (2004). hep-ph/0409146

71. S. Frixione, P. Nason, C. Oleari, JHEP 0711, 070 (2007). arXiv:0709.2092 [hep-ph]

72. S. Alioli, P. Nason, C. Oleari, E. Re, JHEP 1006, 043 (2010). arXiv:1002.2581 [hep-ph]

73. V.M. Abazov et al., D0 Collaboration, Phys. Lett. B 703, 422 (2011). arXiv:1104.2887 [hep-ex]

74. S. Chatrchyan et al., CMS Collaboration, Phys. Lett. B 728, 496 (2014). arXiv:1307.1907 [hep-ex]

75. A. Kharchilava, Phys. Lett. B 476, 73 (2000). hep-ph/9912320

76. G. Corcella, M.L. Mangano, M.H. Seymour, JHEP 0007, 004 (2000). hep-ph/0004179

77. R. Chierici and A. Dierlamm, CMS NOTE 2006/058 (2006).

78. G. Corcella and F. Mescia, Eur. Phys. J. C 65, 171 (2010) [Erratum-ibid. C 68, 687 (2010)] arXiv:0907.5158 [hep-ph]

79. G. Corcella, A.D. Mitov, Nucl. Phys. B 623, 247 (2002). hep-ph/0110319

80. M. Cacciari, G. Corcella, A.D. Mitov, JHEP 0212, 015 (2002). hep-ph/0209204

81. G. Corcella, V. Drollinger, Nucl. Phys. B 730, 82 (2005). hep-ph/0508013

82. ATLAS Collaboration, ATLAS-CONF-2013-077 (2013)

83. S. Frixione, A. Mitov, JHEP 1409, 012 (2014). arXiv:1407.2763 [hep-ph] 
84. S. Chatrchyan et al. [CMS Collaboration], Eur. Phys. J. C 73, 2339 (2013). arXiv:1211.2220 [hep-ex]

85. S. Chatrchyan et al., CMS Collaboration, JHEP 1212, 105 (2012) arXiv:1209.2319 [hep-ex]

86. S. Chatrchyan et al., CMS Collaboration, Eur. Phys. J. C 72, 2202 (2012) arXiv:1209.2393 [hep-ex]

87. S. Chatrchyan et al., CMS Collaboration, Eur. Phys. J. C. 74, 2758 (2014). arXiv:1307.4617 [hep-ex]

88. M. Czakon, P. Fiedler, A. Mitov, Phys. Rev. Lett. 110, 252004 (2013). arXiv:1303.6254 [hep-ph]

89. M. Czakon, A. Mitov, JHEP 1301, 080 (2013). arXiv:1210.6832 [hep-ph]

90. M. Czakon, A. Mitov, JHEP 1212, 054 (2012). arXiv:1207.0236 [hep-ph]

91. P. Baernreuther, M. Czakon, A. Mitov, Phys. Rev. Lett. 109, 132001 (2012). arXiv:1204.5201 [hep-ph]

92. M. Seidel, Top Quark Mass Results at the LHC, 5th International Workshop on Top Quark Physics (Winchester, 2012)

93. CMS and ATLAS Collaborations, ATLAS-CONF-2013-102, CMS PAS TOP-13-005 (2013)

94. S. Chatrchyan et al., CMS Collaboration, Eur. Phys. J. C 73, 2494 (2013) arXIv:1304.5783 [hep-ex]

95. M. Burns, K. Kong, K.T. Matchev, M. Park, JHEP 0903, 143 (2009). arXiv:0810.5576 [hep-ph]

96. A.J. Barr, C.G. Lester, J. Phys. G 37, 123001 (2010). arXiv:1004.2732 [hep-ph]

97. ATLAS Collaboration, ATLAS-CONF-2013-046 (2013)

98. A.D. Martin, W.J. Stirling, R.S. Thorne, G. Watt, Eur. Phys. J. C 63, 189 (2009). arXiv:0901.0002

99. R.D. Ball, V. Bertone, S. Carrazza, C.S. Deans, L. Del Debbio, S. Forte, A. Guffanti, N.P. Hartland et al., Nucl. Phys. B 867, 244 (2013). arXiv:1207.1303 [hep-ph]

100. [DELPHI Collaboration], ICHEP 2002 Note, DELPHI 2002-069 CONF 603

101. M. Czakon, M.L. Mangano, A. Mitov, J. Rojo, JHEP 1307, 167 (2013). arXiv:1303.7215 [hep-ph]

102. S. Alioli, P. Fernandez, J. Fuster, A. Irles, S.-O. Moch, P. Uwer, M. Vos, Eur. Phys. J. C 73, 2438 (2013). arXiv:1303.6415 [hep-ph]

103. J.E. Brau, R.M. Godbole, F.R.L. Diberder, M.A. Thomson, H. Weerts, G. Weiglein, J.D. Wells, H. Yamamoto, arXiv:1210.0202 [hep-ex]

104. P. Lebrun, L. Linssen, A. Lucaci-Timoce, D. Schulte, F. Simon, S. Stapnes, N. Toge and H. Weerts et al., arXiv:1209.2543 [physics.ins-det]

105. M. Bicer et al., TLEP Design Study Working Group Collaboration, JHEP 1401, 164 (2014). arXiv:1308.6176 [hep-ex]

106. J-P. Delahaye, C. Ankenbrandt, A. Bogacz, S. Brice, A. Bross, D. Denisov, E. Eichten, P. Huber et al., arXiv:1308.0494 [physics.acc-ph]

107. J.H. Kuhn, Acta Phys. Polon. B 12, 347 (1981)

108. M. Martinez, R. Miquel, Eur. Phys. J. C 27, 49 (2003). hep-ph/0207315

109. M. Beneke, Y. Kiyo, K. Schuller, PoS RADCOR 2007, 051 (2007). arXiv:0801.3464 [hep-ph]

110. M. Stahlhofen, A. Hoang, PoS RADCOR 2011, 025 (2011). arXiv:1111.4486 [hep-ph]

111. K. Seidel, F. Simon, M. Tesar, S. Poss, Eur. Phys. J. C 73, 2530 (2013). arXiv:1303.3758 [hep-ex]

112. G.T. Bodwin, E. Braaten, G.P. Lepage, Phys. Rev. D 51, 1125 (1995) [Erratum-ibid. D 55, 5853 (1997)]. hep-ph/9407339

113. N. Brambilla, A. Pineda, J. Soto, A. Vairo, Nucl. Phys. B 566, 275 (2000). hep-ph/9907240

114. B.A. Kniehl, A.A. Penin, Nucl. Phys. B 563, 200 (1999). hep-ph/9907489
115. A.A. Penin, M. Steinhauser, Phys. Lett. B 538, 335 (2002). hep-ph/0204290

116. B.A. Kniehl, A.A. Penin, Nucl. Phys. B 577, 197 (2000). hep-ph/9911414

117. B.A. Kniehl, A.A. Penin, V.A. Smirnov, M. Steinhauser, Nucl. Phys. B 635, 357 (2002). hep-ph/0203166

118. B.A. Kniehl, A.A. Penin, M. Steinhauser, V.A. Smirnov, Phys Rev. Lett. 90, 212001 (2003). hep-ph/0210161

119. A.H. Hoang, Phys. Rev. D 69, 034009 (2004). hep-ph/0307376

120. A.A. Penin, V.A. Smirnov, M. Steinhauser, Nucl. Phys. B 716, 303 (2005). hep-ph/0501042

121. M. Beneke, Y. Kiyo, K. Schuller, Nucl. Phys. B 714, 67 (2005). hep-ph/0501289

122. P. Marquard, J.H. Piclum, D. Seidel, M. Steinhauser, Nucl. Phys. B 758, 144 (2006). hep-ph/0607168

123. M. Beneke, Y. Kiyo, K. Schuller, Phys. Lett. B 658, 222 (2008). arXiv:0705.4518 [hep-ph]

124. M. Beneke, Y. Kiyo, A.A. Penin, Phys. Lett. B 653, 53 (2007). arXiv:0706.2733 [hep-ph]

125. M. Beneke, Y. Kiyo, Phys. Lett. B 668, 143 (2008). arXiv:0804.4004 [hep-ph]

126. C. Anzai, Y. Kiyo, Y. Sumino, Phys. Rev. Lett. 104, 112003 (2010). arXiv:0911.4335 [hep-ph]

127. A.V. Smirnov, V.A. Smirnov, M. Steinhauser, Phys. Rev. Lett. 104 112002 (2010). arXiv:0911.4742 [hep-ph]

128. M. Beneke, Y. Kiyo, A. Penin, K. Schuller, eConf C 0705302, TOP01 (2007). arxiv:0710.4236 [hep-ph]

129. Y. Kiyo, Y. Sumino, Phys. Rev. D 67, 071501 (2003). hep-ph/0211299

130. C. Bauer, G.S. Bali, A. Pineda, Phys. Rev. Lett. 108, 242002 (2012). arXiv:1111.3946 [hep-ph]

131. A.H. Hoang, A.V. Manohar, I.W. Stewart, T. Teubner, Phys. Rev. Lett. 86, 1951 (2001). hep-ph/0011254

132. A.A. Penin, A. Pineda, V.A. Smirnov, M. Steinhauser, Nucl. Phys. B 699, 183 (2004). hep-ph/0406175

133. A. Pineda, A. Signer, Nucl. Phys. B 762, 67 (2007). hep-ph/0607239

134. A.H. Hoang, M. Stahlhofen, JHEP 1405, 121 (2014). arXiv:1309.6323 [hep-ph]

135. A.H. Hoang, C.J. Reißer, Phys. Rev. D 71, 074022 (2005). hep-ph/0412258

136. A.H. Hoang, C.J. Reisser, Phys. Rev. D 74, 034002 (2006). hep-ph/0604104

137. T. Nagano, A. Ota, Y. Sumino, Phys. Rev. D 60, 114014 (1999). hep-ph/9903498

138. G. Aarons et al., arXiv:0709.1893 [hep-ph]

139. D. Schulte, Ph.D. Thesis, University of Hamburg, TESLA-97-08 (1996)

140. F. Gournaris, Ph.D. Thesis, University College London (2009)

141. S. Poss, A. Sailer, Eur. Phys. J. C 74, 2833 (2014). arXiv:1309.0372 [physics.ins-det]

142. S. Boogert, M. Hildreth, D. Käfer, J. List, K. Mönig et al., JINST 4, P10015 (2009)

143. A. Hinze, K. Mönig, eConf C050318, 1109 (2005)

144. The ILD Concept Group, arXiv:1006.3396 [hep-ex]

145. The SiD Concept Group, arXiv:0911.0006 [physics.ins-det]

146. K. Seidel, S. Poss, F. Simon, LCD-Note-2011-026 (2011)

147. S.V. Chekanov, Eur. Phys. J. C 26, 13 (2002). hep-ph/0206264

148. V.A. Khoze, T. Sjostrand, Eur. Phys. J. direct C 2, 1 (2000). hep-ph/9912297 